\title{
Les sources de l'histoire du Réseau breton
}

\section{Armelle Le Guyader}

\section{(2penEdition}

\section{Journals}

Édition électronique

URL : https://journals.openedition.org/rhcf/2078

DOI : 10.4000/rhcf.2078

\section{Éditeur}

Rails \& histoire

Édition imprimée

Date de publication : 2 mai 2002

Pagination : 328-341

ISBN : 00996-9403

ISSN : 0996-9403

\section{Référence électronique}

Armelle Le Guyader, «Les sources de l'histoire du Réseau breton », Revue d'histoire des chemins de fer

[En ligne], 24-25 | 2002, mis en ligne le 21 avril 2015, consulté le 22 avril 2022. URL : http:// journals.openedition.org/rhcf/2078 ; DOI : https://doi.org/10.4000/rhcf.2078 


\section{Armelle LE GUYADER}

\section{Les sources de I'histoire du Réseau breton}

La première ligne du Réseau breton, de Morlaix à Carhaix, fut ouverte à l'exploitation le 28 septembre 1891. Après plus de vingt ans de pourparlers entre les dirigeants locaux (les maires des différentes communes laissées pour compte, les conseillers départementaux et régionaux) et l'administration des travaux publics, un réseau ferroviaire commençait à voir le jour en Centre Bretagne*.

La Bretagne disposait déjà de lignes de chemin de fer, mais elles ne desservaient que les départements côtiers et la région rennaise. L'idée d'un réseau breton apparaissait alors comme le moyen de compléter le réseau à voie normale. En effet, par souci d'économie le nouveau réseau fut construit à voie métrique. Cela réduisait le coût de la construction d'ouvrages d'arts (comme les viaducs), tout en permettant de pénétrer dans les vallées et autres endroits quelque peu accidentés auxquels une ligne à voie normale n'aurait pu avoir accès sans recourir à d'importants travaux d'aménagement. Durant l'entre-deux-guerres, l'étoile ferroviaire carhaisienne prit toute son ampleur, et le Réseau breton devint le réseau à voie métrique le plus important de France.

Par l'intermédiaire de ce nouveau moyen de transport, les départements espéraient sortir de l'enclavement dans lequel ils étaient confinés. Grâce à de nouveaux débouchés, ils pensaient dynamiser leur économie et permettre à la population de bénéficier de meilleurs moyens de communication (le réseau routier étant durant certaines saisons impraticable). L'amélioration des conditions de vie des Bretons devait venir grâce au chemin de fer. L'économie locale, essentiellement agricole comme dans beaucoup de régions à cette époque, découvrit un moyen d'échange et d'exportation important. La circulation du bétail (chevaux, bovins et porcins entre autres) s'intensifia et le ravitaillement des agriculteurs en engrais marin en provenance de Morlaix ou Camaret fut plus efficace. De nombreux emplois directs ou induits furent créés.

\footnotetext{
* Ces pages sont extraites de l'introduction et du premier chapitre du mémoire de maîtrise de Mlle Le Guyader,Carbaix, une étoile ferroviaire en Centre Bretagne, 1891 1938, préparé en 2000-2001 à l'université de Haute-Bretagne Rennes II sous la direction de Mme Anne-Françoise Garçon et avec le soutien de l'AHICF.
} 
Mais le chemin de fer participa également à une certaine modernisation des campagnes. Il stimula de nouveaux aménagements comme l'électrification. Des services apparurent ou se développèrent (les envois postaux étaient désormais plus rapides). Bref, la population bénéficia de nouvelles facilités. Le Réseau breton fut achevé en 1926, et prit la forme d'un réseau étoilé dont le centre était situé à Carhaix dans le Finistère. De 1891 à 1938, date à laquelle la SNCF reprit en main le réseau, il connut une époque très florissante. Désormais, l'Armor (le pays de la mer) et l'Argoat (le pays de la forêt) étaient directement reliés. De Carhaix, on pouvait joindre : Morlaix, Guingamp, Paimpol, Loudéac, La Brohinière, Rosporden, Châteaulin, Camaret et Le Fret. Quatre départements étaient donc traversés. Cette nouveauté en Centre Bretagne était le résultat d'une longue lutte entre l'État et les dirigeants locaux.

La Bretagne intérieure fait partie de ces régions qui ont eu du mal à se doter d'un réseau de chemin de fer. Seules les régions côtières étaient desservies. Le plan Freycinet ne reconnaissait pas à la liaison entre la côte et les terres une impérieuse nécessité. Ce sont donc les pouvoirs locaux qui se chargèrent de prouver l'intérêt que présentait cette liaison. En 1865, les pouvoirs publics avaient pris les devants en préconisant la construction d'un chemin de fer à voie métrique. L'administration des travaux publics prenait à sa charge la moitié des dépenses, à charge pour la région de mettre le Réseau breton à voie normale par la suite. En 1883, la Compagnie de l'Ouest obtint la concession des premières lignes du réseau mais ce fut la Société des chemins de fer économiques qui fut chargée par elle de l'exploitation. Le traité d'affermage fut signé le 5 mars 1886. Cette convention fut reconduite en 1909 lors du rachat de la Compagnie de l'Ouest par l'État, puis par la SNCF en 1938 lors de la fusion des anciennes compagnies.

Les enquêtes d'utilité publique réalisées auprès de la population montrèrent l'enthousiasme des communes face à ce projet, notamment en ce qui concerne le choix des tracés. Chaque commune désirait profiter du train et pour cela il fallait, de préférence, que ce dernier passât sur son territoire ou du moins à proximité. Les intérêts économiques et stratégiques étaient les principaux arguments mis en avant par les communes afin de convaincre le ministre des Travaux publics et les ingénieurs du dynamisme de leur territoire. Toutes ces démarches retardèrent d'autant le commencement des travaux.

En dehors du point de vue économique, peu d'études ont été réalisées sur la manière dont le Réseau breton a interféré dans la vie quotidienne des populations locales. Lorsque l'on se rend en Bretagne de nos jours, les personnes qui ont connu ces lignes en parlent avec une 
certaine nostalgie alors que, dans bien des cas, ils n'ont pris le train que peu de fois ou très tardivement. Dans la commune de Gourin, située à une vingtaine de kilomètres de Carhaix, il y avait deux gares : celle du Réseau breton et celle de la Compagnie de l'Ouest qui menait à Lorient. Paradoxalement, le train qui se rendait à Lorient était appelé « le petit train » tandis que la gare du Réseau breton correspondait à « la grande gare ». Cette anecdote montre l'impact qu'eut le chemin de fer et, en particulier, l'étoile ferroviaire de Carhaix, sur les populations locales.

De 1891 à 1938, le R.B. connut une croissance très importante. Si le trafic voyageurs était relativement faible (par rapport à la population concernée), le trafic marchandises quant à lui ne cessait d'augmenter. De nombreuses communes ainsi que des particuliers demandaient fréquemment une augmentation du nombre de trains ou un changement des horaires afin d'améliorer la circulation des personnes et les relations avec les autres réseaux. Le chemin de fer était très sollicité mais les bouleversements qu'il engendrait créèrent également de nombreux conflits. De tels travaux impliquaient une modification du paysage et, parfois, une restructuration des propriétés ce qui n'était pas pour plaire à tout le monde surtout lorsque les exploitations agricoles étaient directement touchées. Les pétitions, les réclamations sont donc nombreuses dans les différentes archives départementales. Les populations s'exprimaient assez facilement sur le problème car pour la plupart (en tant que cultivateurs) leur enrichissement ainsi que l'amélioration de leur vie dépendait de ce nouveau moyen de communication. Les problèmes étaient gérés d'une manière relativement lente car, si l'État était juridiquement responsable, la Société des chemins de fer économiques l'était d'un point de vue administratif et, enfin, les communes et le conseil général étaient les porte-parole de leurs administrés. De plus, l'intérêt des populations locales n'était pas toujours le principal souci des dirigeants.

Les sources de cette étude proviennent essentiellement des archives départementales des Côtes-d'Armor et du Finistère ainsi que des archives privées du Réseau breton. Ces dernières ont été transférées de Morlaix à Carhaix après la fermeture des lignes autres que carhaix-Paimpol en 1967 et sont constituées des dossiers du personnel de la Société des chemins de fer économiques depuis 1891. Les documents trouvés m’ont permis de mieux comprendre l'importance du R.B. et des changements qu'il a apportés en Centre Bretagne. Par contre, ils ne m’ont pas renseignée de manière précise sur le trafic du réseau à cette époque. Les ouvrages consacrés essentiellement au R.B. sont généralement axés sur la période d'après guerre car les éléments d'informations concernant cette période sont beaucoup plus nombreux. Néanmoins, ils m’ont aidée à comprendre le fonctionnement du réseau, notamment sa gestion. 


\section{Présentation des sources}

Plusieurs livres sur le Réseau breton ont été publiés mais peu sont consacrés aux premières années de l'exploitation, parce que les documents dont nous disposons pour traiter cette période sont beaucoup moins nombreux. De plus, toutes les archives du R.B. ne sont pas classées et ne sont pas répertoriées aux Archives départementales.

\section{Les archives départementales}

Quatre départements étaient traversés par le Réseau breton, quatre centres d'Archives départementales étaient donc à étudier. Mais rapidement $j$ 'ai pu constater un déséquilibre dans la masse respective des informations fournies (Annexe 1).

Les archives d'Ille-et-Vilaine ne disposent que de peu de renseignements sur ce sujet et beaucoup d'affaires ne sont pas complètes. Les archives des Côtes-d'Armor et du Finistère sont quant à elles très fournies. Outre les documents relatifs aux avant-projets et aux études de tracé des lignes, les plaintes de la population, les réclamations des communes et des chambres de commerce sont également à notre disposition. Ainsi, il est possible de mesurer l'importance du réseau d'un point de vue administratif mais aussi de mesurer l'impact que sa construction et sa mise en service ont eu sur la population locale. Les séries $41 \mathrm{~s}$ à $47 \mathrm{~s}$ des Côtes-d'Armor et les séries $5 \mathrm{~s} 70$ à $5 \mathrm{~s} 81$ du Finistère sont exclusivement réservées au réseau et plus précisément à sa construction et à son exploitation. Mais de nombreux documents manquent. Dans certains cas nous ne disposons pas de tous les éléments d'une affaire. Parfois, le flou est tel qu'il n'est pas possible de reconstituer le problème et donc des interrogations subsistent. Ainsi, le $1^{\text {er }}$ avril 1892, le conseil municipal de Grâces adressa au sous-préfet de Guingamp une requête concernant une demande de halte sur le territoire de leur commune mais la réponse du sous-préfet ne figure pas dans le dossier ${ }^{1}$.

D’une manière générale, les documents sont classés par affaire et par ligne. Ce classement nous permet d'ailleurs de mieux nous rendre compte de la lenteur administrative et juridique pour la moindre réclamation soulevée. Plusieurs années étaient parfois nécessaires avant que des travaux ou des modifications ne soient exécutées. En 1909, le conseil municipal de Grâces fit une demande pour la construction d'une patte d'oie en Grâces-Guingamp. Ce n'est que le 8 mars 1949 que l'affaire fut classée avec le refus de l'ingénieur chef d'exploitation du R.B. ${ }^{2}$

L'évolution de chaque ligne est également plus facile à percevoir. On se rend compte de l'importance du trafic et donc de la viabilité et de la rentabilité d'un tel service. Les archives du Finistère concernent 
surtout les lignes de Châteaulin à Carhaix, de Carhaix à Rosporden, de Carhaix à Morlaix et de Châteaulin à Camaret-sur-Mer. Les lignes allant de Carhaix à Guingamp, de Guingamp à Paimpol, de la Brohinière à Loudéac et de Loudéac à Carhaix sont détaillées aux archives des Côtesd'Armor. A partir de ces deux centres, il est donc possible de trouver des renseignements sur toutes les lignes du réseau. Cependant, il n'existe que peu de documents nous permettant d'avoir des données chiffrées sur le trafic. Il n'est pas possible de donner le tonnage transporté sur les lignes ni le nombre de personnes ayant pris le train, que ce soit sur une année ou sur l'ensemble de la période étudiée. Les seuls chiffres qui se trouvent à notre disposition correspondent à des évaluations réalisées au moment des avant-projets et des études. Mais là, encore, peu de ces évaluations subsistent ${ }^{3}$. Aussi, afin de palier ce manque de données, on peut étudier l'évolution architecturale des gares. L'agrandissement des quais, des bâtiments pour voyageurs sont autant de renseignements qui nous donnent des indications sur la fréquentation des lignes. Les demandes de trains supplémentaires sont également des indices.

En dehors de la série $S$, la série $M$ des archives du Finistère ainsi que les nombreux journaux de l'époque m'ont permis de trouver des informations qui se démarquaient du contenu des documents administratifs.

Les séries $8 \mathrm{M}$ et $10 \mathrm{M}$ correspondent aux rapports de la chambre de commerce de Morlaix et aux mouvements de grèves qui ont touché le R.B. De cette manière, on peut plus facilement connaître les réactions de la population ainsi que les intérêts économiques défendus. Les grèves représentent un moyen d'expression, de contestation. Or, même si elles étaient principalement dues aux conditions de travail, sans remettre en cause l'existence du réseau, elles perturbèrent le trafic. C'est dans de pareilles situations que l'on se rend compte de l'importance du service proposé et, en l'occurrence, de l'importance du R.B. et de ses relations avec l'ensemble de la région.

Les journaux transmettent une image populaire du chemin de fer. Les événements relatés correspondent principalement aux inaugurations. Les cérémonies y sont contées de manière très minutieuse, comme le voulait le style de l'époque, ce qui nous permet d'en connaitre les moindres détails. Le nom des personnalités, leur discours, la manière dont s'est déroulée la cérémonie, la présence de la foule constituent autant de moyens permettant de mesurer l'ampleur de l'événement. A part ces occasions, la presse était très peu utilisée par le Réseau breton. La publicité, contrairement à la Compagnie de l'Ouest, ne semblait pas une priorité. De plus, seuls les journaux locaux apportaient des 
renseignements. Je n'ai trouve que peu d'allusions dans l'Ouest Éclair. Le Finistêre, Le Petit Brestois, La dépêche de Brest, Le Petit Libéral ou Le Journal de Guingamp ont été mes principales références. Pour ce qui est des événements particuliers comme les pèlerinages, $j$ 'ai cherché des informations dans : La Semaine religieuse du diocèse de Léon et de Quimper, mais seule la Compagnie de l'Ouest passait des annonces promotionnelles concernant ces fêtes religieuses qui pouvaient concerner ses lignes du littoral.

Les archives départementales sont donc une source importante dans l'étude du Réseau breton, mais elles ne permettent pas d'aboutir à une évaluation chiffrée. Elles ne fournissent pas de renseignements sur les employés du R.B. non plus ainsi que sur le fonctionnement du réseau. Les archives privées du réseau, conservées à Carhaix me permirent ainsi d'élargir mes connaissances sur le sujet.

\section{Les archives privées du Réseau breton}

Si Carhaix était le centre géographique du R.B. et, donc, la plaque tournante du réseau, Morlaix en était le centre administratif. Après la fermeture du réseau, l'ensemble des documents fut transféré à Carhaix qui est désormais le centre des archives privées du Réseau breton et la tête de la ligne à voie normale Carhaix-Paimpol aujourd'hui exploitée par la C.F.T.A. ${ }^{4}$. Cependant, mis à part les dossiers du personnel depuis 1891, aucun document (administratif) n'y figure. Ces dossiers sont une source d'informations très importante. Ils sont classés par année et dans l'ordre d'entrée des agents. Malheureusement, l'essentiel des dossiers stockés à Carhaix concerne des agents ayant travaillé à Carhaix ou à proximité. Un grand nombre de documents a du être perdu dans le déménagement des archives.

La masse d'informations contenue dans les dossiers est très importante et, par manque de temps, j'ai choisi d'étudier seulement quelques années, à savoir 1895, 1898, 1903, 1909, 1915, 1919, 1923, 1929, 1933, 1938. Chaque dossier comprenait (dans l'ordre qui suit) : les papiers certifiant le départ de l'employé (c'est-à-dire, suivant les cas : lettre de démission, certificat de décès, départ en retraite, mutation ou encore lettre de licenciement) ; les relevés des états de service ; les amendes ou les gratifications délivrées ; le bulletin de naissance des enfants ; l'extrait de mariage (surtout après les années 1920) ; l'extrait du casier judiciaire ; un certificat de bonne vie et de mœurs ; un certificat médical (médecin de la Société générale des chemins de fer économiques, la S.E.) ; le procès-verbal d'examen et, enfin, la lettre de demande d'emploi. La moindre maladie, les jours d'absence (et leurs motifs), la participation aux mouvements de grève, le rattachement à un syndicat, bref, tout 
était connu et répertorié. Quelques modifications apparaissent dans la constitution des dossiers au fur et à mesure des décennies. Le niveau d'examen (« le procès-verbal d'examen »), était un peu plus élevé vers 1930, mais le principe restait le même : un peu d'algèbre (addition, soustraction, division et multiplication) puis une dictée qui en général correspondait à un extrait du règlement de la Société des chemins de fer économiques ou à des règles de sécurité à respecter aux abords des voies de chemin de fer.

La première page de chaque dossier retrace l'ensemble de la carrière de chaque employé avant, pendant et après sa sortie de la compagnie. Dans le cas de M. Minoux Yves (Annexe 2), on apprend dans un premier temps qu'il fut employé au service de la traction (en haut à droite) puis qu'il est né le 9 janvier 1864 à Plouëz, qu'il travaillait auparavant en tant que maçon à Guingamp et qu'il savait lire, écrire et qu'il connaissait les quatre règles. Étant donné le niveau d'instruction et la profession antérieure de M. Minoux, il ne semblait pas y avoir de qualification spécifique requise pour entrer au R.B. puisqu'il fut nommé au grade de chauffeur de troisième classe. Sur l'ensemble des dossiers étudiés le niveau d'instruction est identique à celui-ci et les activités professionnelles antérieures sont très diverses. La situation militaire est également indiquée ce qui permettait à la société de connaitre le nombre de ses agents susceptibles de mobilisation. De plus, cela évitait d'employer un déserteur et donc d'être en règle. Le tableau qui suit retrace la carrière de l'agent au sein même de la société ainsi que sa situation familiale. Yves Minoux n'est pas resté longtemps au R.B., il y est entré le 10 août 1894 et en est parti le 13 août 1895. Sa courte carrière ne lui a pas permis de progresser en grade et donc son salaire est resté identique. Par contre, il a subi un changement d'affectation, trois mois après son arrivée à Carhaix il se retrouva à Paimpol, le 9 octobre. Enfin, un dernier tableau nous renseigne sur les raisons de son départ et sur les observations de son employeur. Toutes les personnes étaient ainsi répertoriées.

Par ailleurs, il existait un registre du personnel avec le relevé de tous les employé depuis l'ouverture de la première ligne du réseau et du poste occupé. On découvre ainsi que beaucoup de femmes étaient employées. Il existait une multitude de professions, j’en ai relevé 38 allant du statut de lingère à celui d'inspecteur attaché au chef d'exploitation.

Ce registre m'a permis de me rendre compte qu'il manquait un certain nombre de dossiers. De plus, ces derniers ne concernent que les employés ayant travaillé à Carhaix ou rattachés à Carhaix. Lors du déménagement des archives de Morlaix à Carhaix, il dut y avoir des pertes ou bon nombre d'entre elles sont restées à Morlaix. 
R.B. $[\ldots]^{5}$.

Je n'ai trouvé aucun document faisant référence au trafic du

Néanmoins, les archives du Réseau breton sont une source de renseignements considérable, notamment sur les conditions de travail de l'époque, et elles mériteraient à elles seules un travail complet. Aucun des livres que j'ai étudiés n'y fait référence, tout comme ils ne font pas référence de manière précise au trafic marchandises et voyageurs de cette époque.

\section{Notes}

1- AD des Côtes-d'Armor série 42s4, ligne Carhaix-Guingamp, lettre du conseil municipal de Grâces au sous-préfet de Guingamp, le $1^{\text {er }}$ avril 1892.

2- AD des Côtes-d'Armor série 42s6, ligne Carhaix-Guingamp, lettre de l'ingénieur chef d'exploitation au sous-préfet de Guingamp, le 8 mars 1949.

3- Mlle Le Guyader n'a pu avoir accès aux Statistiques annuelles du ministère des Travaux publics dont nous reproduisons un exemple (Annexe 3). Les Rapports annuels de la Société des chemins de fer économiques peuvent également être consultés, dans une collection privée (N.d.l.R.).

4- Ces archives, à la suite du travail effectué par Mlle Le Guyader, sont en cours de versement dans les archives publiques à l'initiative de l'agence régionale de la C.F.T.A. (N.d.l.R.).

5- Voir note 3 et Annexe 3 (N.d.l.R.). 


\section{Annexe 1}

\section{Les sources de l'histoire du Réseau breton dans les Archives départementales}

\section{. Les archives départementales des Côtes d'Armor}

\section{Les sources manuscrites}

Ligne Carhaix-Morlaix :

Série 41s 1 : Acquisition et construction

Série 41s2 : Etablissements des stations, enquêtes parcellaires.

Série 41s3 : Occupation temporaire, enquêtes parcellaires.

Série 41s 4 : Marche des trains, amélioration du profil en long, exploitation.

Ligne Carhaix-Guingamp :

Série 42s1 : Construction, acquisition, stations.

Série 42s2 : Tracé.

Série 42s3 : Travaux, états parcellaires, ouvrages d'art, service.

Série 42s 4 : Réclamations, contestations.

Série $42 s 5$ : Réclamations, contestations.

Série 42s6 : Réclamations, contestations.

Ligne Guingamp-Paimpol :

Série 43s1 : Demandes d'alignements.

Série 43s2 : Réclamations, indemnités.

Série 43s3 : Travaux.

Série 43s5: Marche des trains.

Série $43 \mathrm{~s} 6$ : Sécurité, trafic, marche des trains, commerce.

Ligne St Méen-Loudéac-Carhaix :

Série 45s1 : Autorisation ouverture de commerce.

Série 45s 2 : Délibération, construction.

Série 45s 3 : Exécution des travaux, vœux des communes et des particuliers.

Série 45s 4 : Acquisition de terrains, expropriations, haltes, vœux.

Série 45s5 : Sécurité, horaires, marche des trains.

Série 45s6 : Sécurité, vœux.

Ligne de La Brohinnière à la ligne de Châteaulin à Landerneau par Loudéac et Carhaix (première section) :

Série $47 \mathrm{~s} 1$ : Autorisation de construction.

Série $47 \mathrm{~s} 2$ : Délibérations des tracés, des stations.

Série $47 \mathrm{~s} 3$ : Réclamations, ouvrages d'art. 
Série 47s 4 : Réclamations, acquisitions, expropriations.

Série 47s5: Marche des trains, réclamations.

Série $47 \mathrm{~s} 6$ : Réclamations, plaintes.

Série S supplément 17 : Documents divers non classés sur l'ensemble des lignes traversant le département: Procès verbaux, marche des trains, autorisation, travaux, accords entre les compagnies, autorisation pour la construction des voies.

\section{Les sources imprimées}

Le Petit Libéral:

Série 4mi11-R9 : jeudi 19 mai 1898.

Série 4mi11-R11 : jeudi 14 août 1902.

Série 4mi11-R12 : jeudi 8 août 1907.

Le Journal de Guingamp:

Série 4mi16-R12 : Samedi 23 septembre 1893 ; Samedi 30 septembre 1893.

\section{. Les archives départementales du Finistère}

\section{Les sources manuscrites}

Ligne Châteaulin-Carhaix :

Série 5 s70 : Demande de création de cette ligne, étude avant projet, enquête d'utilité publique.

Ligne Carhaix-Rosporden :

Série 5s71 : Avant-projet, Station, ouverture ligne, concession à la

Compagnie de l'Ouest, vœux, dédommagement.

Ligne Carhaix-Morlaix :

Série $5 \mathrm{~s} 73$ : Autorisation, construction, réclamations, enquêtes d'utilité publique, enquêtes parcellaires.

Série 5874 : Acquisition.

Série 5s75: Halte, construction, embranchement du port, marche des trains, suppression de ligne.

Série 5s76 : Embranchement Morlaix-port-gare : réclamations et construction.

Ligne Carhaix-Châteaulin :

Série 5s78: Demandes de stations, réclamations, trafic.

Ligne Châteaulin-Camaret :

Série 5s79 : Avant-projet, étude du trafic, stations, pétitions.

Ligne Châteaulin-Camaret sur Mer :

Série 5s81 : Demandes de construction, rapports des ingénieurs, évaluations du trafic. 
Mouvements de grèves :

Série 10m23: Création du syndicat du Réseau Breton.

Série 10m48 : Grève des employés de la S.E. à Carhaix.

Tourisme: Liste des hôtels et des restaurants :

Série 3j38-5 : Ville de Crozon.

Série 3j38-12 : Ville de Morlaix.

Série 3j38-4 : Villes de Carhaix, Châteaulin, Châteauneuf du Faou.

\section{Les sources imprimées}

Compte-rendu du conseil général :

Série 1n219 : Séance du 28 juillet 1906 ; séance du 24 août 1906.

Le Courrier du Finistère :

Série 4mi20-6 : samedi $1^{\text {er }}$ août 1896 ; samedi 15 août 1896.

Série 4mi20- 7 : samedi 21 mai 1898 ; samedi 14 avri11899.

Série 4mi20-16 : samedi 13 juin 1925.

Le Finistère :

Série 4mi21-15 : samedi 19 septembre 1891 ; mardi 29 septembre 1891.

Série 4mi21-20 : jeudi 30 juillet 1896.

La dépêche de Brest:

Série 4mi101-37: 7 avri11904.

Série 4mi101-38 : 30 août 1904.

La semaine religieuse du diocèse de Léon et de Quimper:

Série 4mi44-35 : vendredi 06 octobre 1933.

\section{. Les archives privées du Réseau Breton}

Lieu : Carhaix, dans les locaux de la CFTA, ancienne gare du Réseau Breton. Nature des documents : dossiers du personnel.

Masse des documents : depuis 1891 à nos jours.

Années étudiées : 1895, 1898, 1903, 1909, 1915, 1919, 1923, 1929, 1933, 1938. 
Annexe 2

Première page du dossier d'Yves Minoux, extrait.

Source : archives privées du Réseau breton, C.F.T.A., Carhaix.

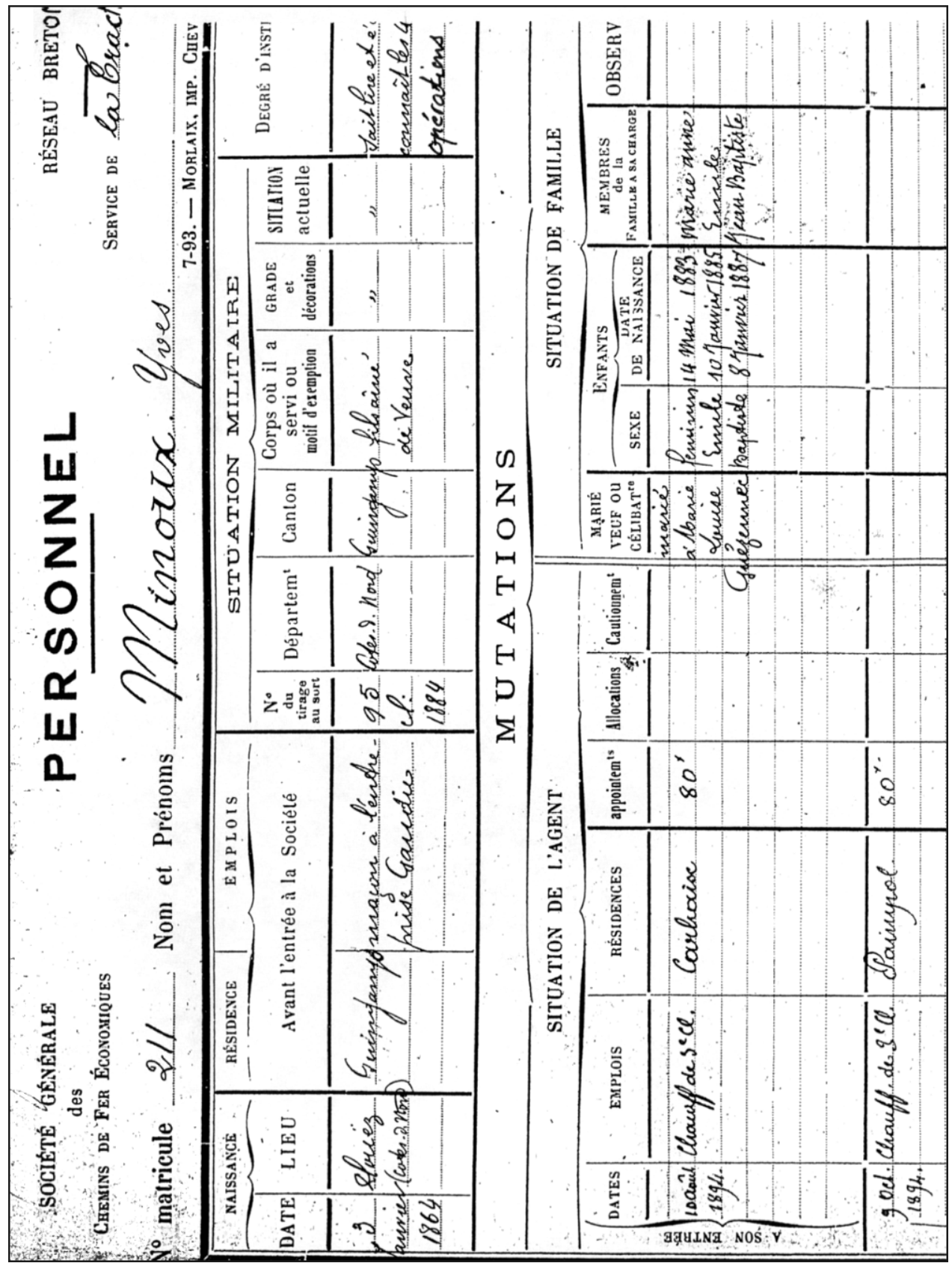


Revue d'histoire des chemins de fer 24-25 (printemps - automne 2001)

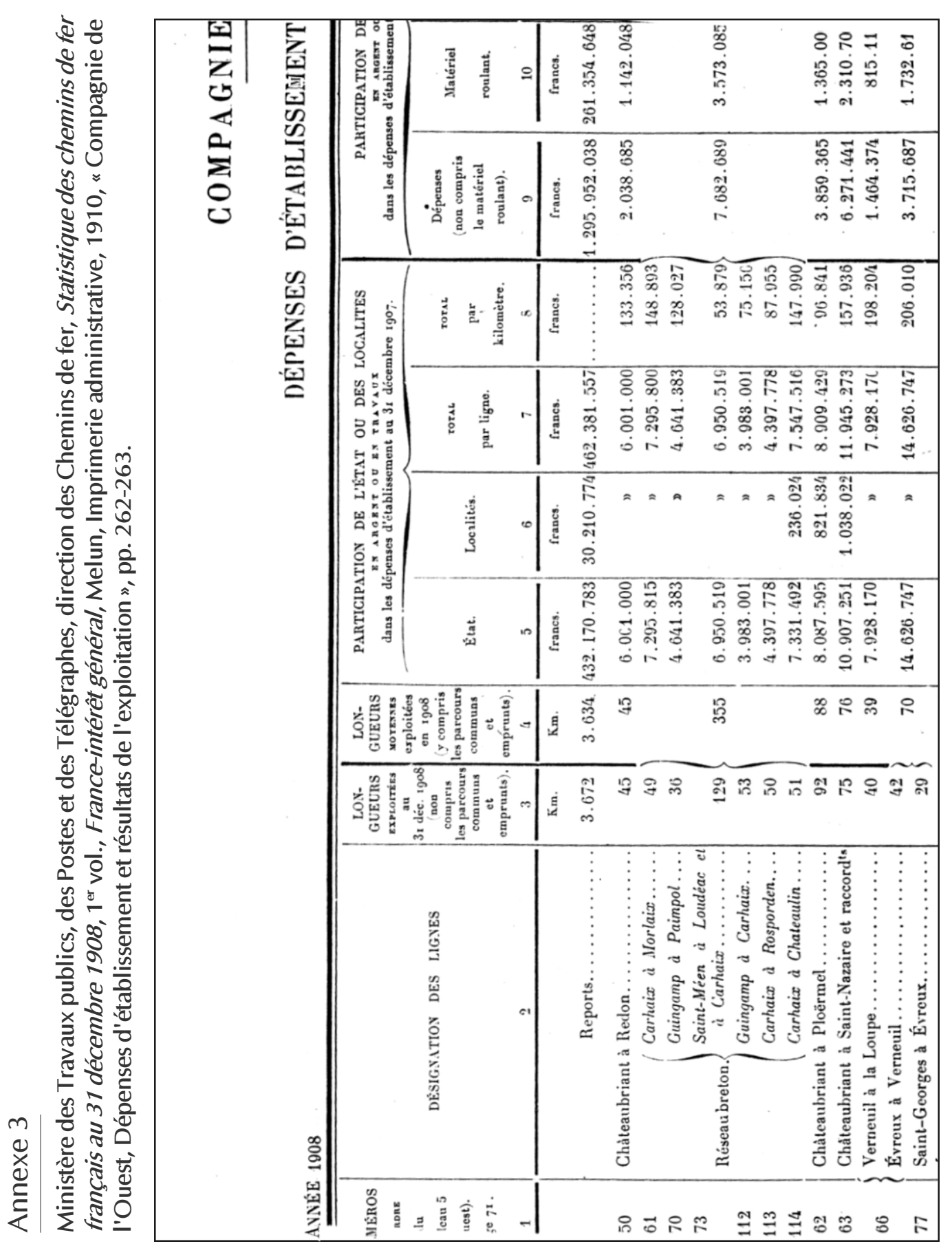




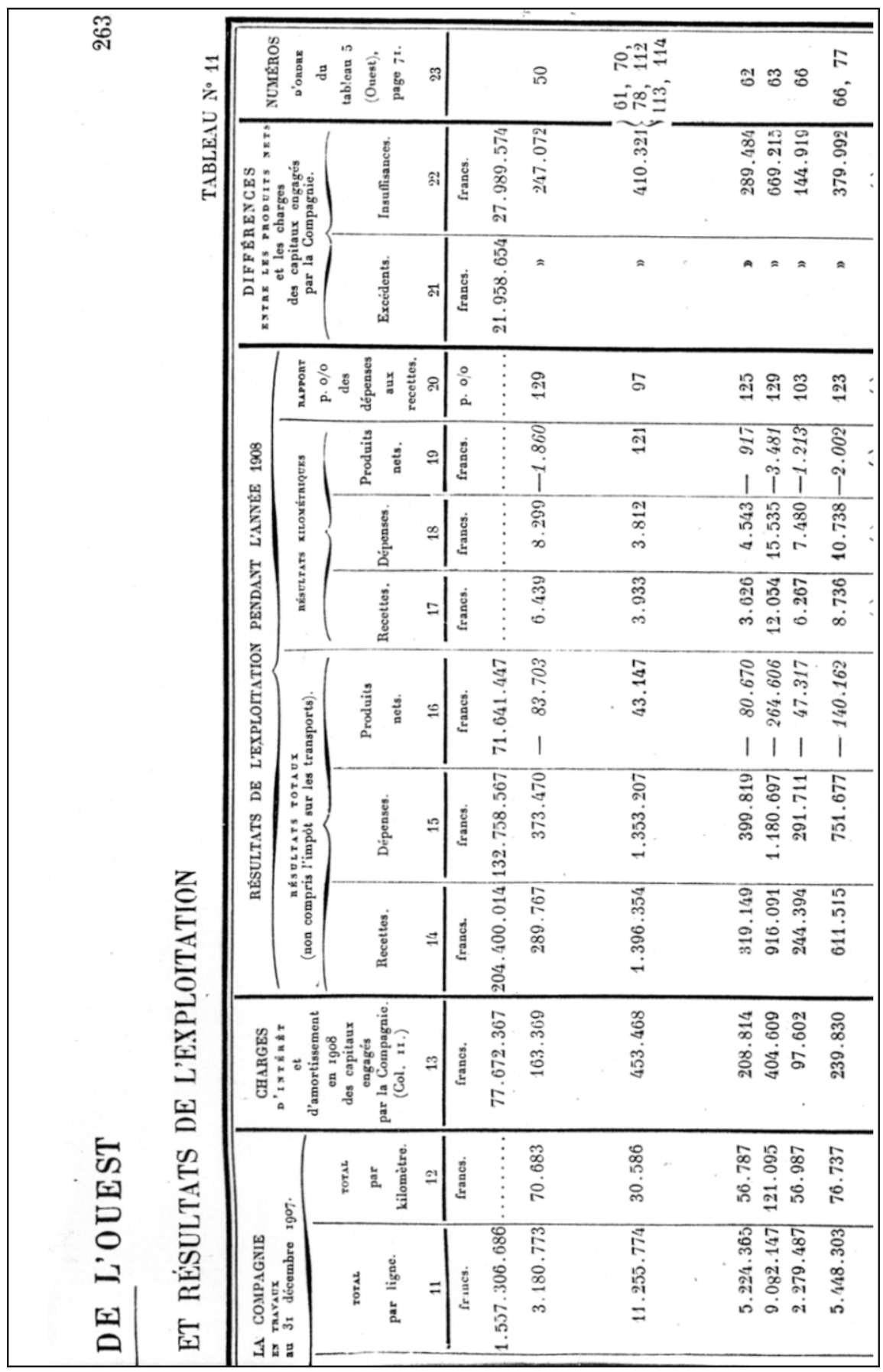

